\title{
Inflation and the semiclassical dynamics of a conformal scalar field
}

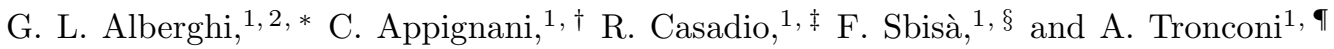 \\ ${ }^{1}$ Dipartimento di Fisica, Università di Bologna, and I.N.F.N., \\ Sezione di Bologna, via Irnerio 46, 40126 Bologna, Italy \\ ${ }^{2}$ Dipartimento di Astronomia, Università di Bologna, via Ranzani 1, 40127 Bologna, Italy.
}

\begin{abstract}
We derive the semiclassical evolution of massless conformally coupled scalar matter in the de Sitter space-time from the Born-Oppenheimer reduction of the Wheeler-DeWitt equation. We find a remarkable difference with respect to the minimally coupled case: the effect of the quantum gravitational corrections do not depend on the momentum of the scalar mode up to second order in the Planck length and, therefore, there are no relevant corrections to the dispersion relation.
\end{abstract}

PACS numbers: 04.60.Kz,04.60.Ds,04.62.+v,98.08.Hw

\section{INTRODUCTION}

Inflation [1] has by now become the standard picture of the early Universe since it solves some of the problems of the standard big-bang scenario and allows one to make testable predictions about the spectrum of the cosmic microwave background radiation. It also provides a window towards trans-Planckian physics [2] as it magnifies all quantum fluctuations and red-shifts originally transPlanckian frequencies down to the range of low energy physics currently observed.

In general, one expects that the standard results of quantum field theory can be no more fully trusted on approaching the Planckian regime or, at least that the dispersion relations of matter fields change for large wavenumber $k$. Most attempts have tested the effects of dispersion relations $\omega=\omega(k)$ chosen ad hoc [2]. In Ref. [3], we have instead derived the dispersion relation for a minimally coupled massless scalar field from a minisuperspace action by lifting the principle of timereparameterization invariance to a quantum symmetry. We then obtained an Hamiltonian constraint from which the Born-Oppenheimer (BO) reduction [4] allowed to properly and unambiguously recover the semiclassical limit of quantum field theory on a curved background starting from the Wheeler-DeWitt (WDW) equation [5]. This procedure yields "quantum fluctuation" terms in the matter equation whose effect on the power spectrum was derived for the simplest model of de Sitter inflation.

In the present paper, we wish to extend the analysis to the case of a generic coupling between the matter field and gravity and will also include an inflaton field so as to consider a generic inflationary evolution, at least for the more formal part. We shall first show that it is possible to treat both the cosmological scale factor $a$ and the inflaton semiclassically, and obtain the relevant corrections for

\footnotetext{
*Electronic address: alberghi@bo.infn.it

${ }^{\dagger}$ Electronic address: appignani@bo.infn.it

¥Electronic address: casadio@bo.infn.it

$\S$ Electronic address: Fulvio.Sbisa@gmail.com

"Electronic address: tronconi@bo.infn.it
}

the matter field. We shall then specialise again to de Sitter inflation, this time for conformally coupled massless scalar matter and derive its effective dynamics. Quite remarkably, we shall see that no relevant corrections appear at (relatively) large scale factor in the Schrödinger equation and the dispersion relation remains unaffected, $\omega \simeq k / a$, to second order in the Planck length. Of course, it is well known that a conformally coupled scalar field can be rescaled and, by making use of the conformal time, it decouples from background gravity (i.e., to zero order in the Planck length). However, our approach allows us to show that, in a sense, this decoupling extends to include quantum gravitational fluctuations to second order in the Planck length. The effect of such fluctuations is given in terms of complicated operators acting on the scalar field state and the "triviality" of the final result is therefore not at all obvious from the outset.

In the next Section, we shall briefly introduce the model and its classical dynamics. For the sake of generality, we shall treat the inflaton as an independent degree of freedom minimally coupled to the cosmological scale factor and also consider a generic coupling of the perturbation field to gravity. The WDW equation in the BO decomposition will then be analysed in Section III and its application to the specific case of a conformally coupled perturbation in de Sitter inflation given in Section IV. Finally, we shall comment on our findings in Section $\mathrm{V}$.

We shall use units with $c=1$ and $\kappa=8 \pi G_{\mathrm{N}}$.

\section{CLASSICAL MODEL}

Let us begin by briefly reviewing the classical dynamics for the system in consideration. More details can be found, for example, in Ref. [6], where however only one matter field was considered.

We start from the action

$$
\begin{aligned}
S=\frac{1}{2} \int \mathrm{d}^{4} x \sqrt{-g}[ & \left(\frac{1}{\kappa}-\xi \Phi^{2}\right) R-(\partial \Phi)^{2}-\mu^{2} \Phi^{2} \\
& \left.-(\partial \Psi)^{2}-m^{2} \Psi^{2}-V(\Psi)\right],
\end{aligned}
$$

where $\Psi$ is the minimally coupled inflaton, $\Phi$ describes 
perturbations with a generic coupling $\xi$ to gravity, $m$ and $\mu$ are the mass of $\Psi$ and $\Phi$ respectively, $g$ is the determinant of the metric and $V(\Psi)$ a potential for $\Psi$. Given the FRW metric [7]

$$
\mathrm{d} s^{2}=-N^{2} \mathrm{~d} t^{2}+a^{2}\left(\frac{\mathrm{d} r}{1-K r^{2}}+r^{2} \mathrm{~d} \Omega^{2}\right)
$$

where $N=N(t)$ is the lapse function and $a=a(t)$ the scale factor, the curvature scalar becomes

$$
R=\frac{6}{N^{2}}\left(\frac{\ddot{a}}{a}-\frac{\dot{a}}{a} \frac{\dot{N}}{N}+\frac{\dot{a}^{2}}{a^{2}}+N^{2} \frac{K}{a^{2}}\right)
$$

where $K=0, \pm 1$ is the spatial curvature and $\dot{f}=\partial_{t} f$.

We are primarily interested in considering the field $\Phi$ as a perturbation over the background driven by the inflaton $\Psi$. Denoting with $\phi_{k}$ a given mode of $\Phi$ and with $\varphi$ the homogenous mode of $\Psi$, the above expression can therefore be written as the sum over the actions for each mode of $\Phi$,

$$
S_{k}=\frac{1}{2} \int N a^{3} \mathrm{~d} t\left[\frac{\dot{\phi}_{k}^{2}}{N^{2}}-\omega_{k}^{2} \phi_{k}^{2}-\frac{6}{a^{2}}\left(v-\xi \phi_{k}^{2}\right)\left(\frac{\dot{a}^{2}}{N^{2}}-K\right)+12 \xi \frac{\phi_{k} \dot{a} \dot{\phi}_{k}}{a N^{2}}+\frac{\dot{\varphi}^{2}}{N^{2}}-m^{2} \varphi^{2}-V_{\varphi}\right]
$$

in which we eliminated second derivatives of $a$ by integrating by parts [6],

$$
\omega_{k}^{2}=\frac{k^{2}}{a^{2}}+\mu^{2}
$$

and $v=\mathcal{V} / \kappa$ where $\mathcal{V} a^{3}$ is the "volume of the universe". In the following, we shall just consider one mode at a time and omit the corresponding index $k$ when this does not cause confusion.

\section{A. Lagrangean dynamics}

Varying the action and then setting $N=1$ (proper time gauge $t=\tau$, so that $\dot{f}=\partial_{\tau} f$ ) yields

$$
\begin{aligned}
\frac{1}{a^{3}} \frac{\delta S}{\delta N}= & 3\left(v-\xi \phi^{2}\right)\left(\frac{\dot{a}^{2}}{a^{2}}+\frac{K}{a^{2}}\right)-\frac{1}{2}\left(\dot{\phi}^{2}+\omega^{2} \phi^{2}\right) \\
& -6 \xi \frac{\dot{a}}{a} \phi \dot{\phi}-\frac{1}{2}\left(\dot{\varphi}^{2}+m^{2} \varphi^{2}+V_{\varphi}\right) \\
= & -\frac{H}{a^{3}}=0 \\
\frac{1}{a^{2}} \frac{\delta S}{\delta a}= & 3\left\{\left(v-\xi \phi^{2}\right)\left(2 \frac{\ddot{a}}{a}+\frac{\dot{a}^{2}}{a^{2}}+\frac{K}{a^{2}}\right)-4 \xi \frac{\dot{a}}{a} \phi \dot{\phi}\right. \\
& -2 \xi\left(\dot{\phi}^{2}+\phi \ddot{\phi}\right)+\frac{1}{2}\left(\dot{\phi}^{2}-\omega^{2} \phi^{2}\right)+\frac{k^{2} \phi^{2}}{3 a^{2}} \\
& \left.+\frac{1}{2}\left(\dot{\varphi}^{2}-m^{2} \varphi^{2}-V_{\varphi}\right)\right\}=0
\end{aligned}
$$

$\begin{aligned} \frac{1}{a^{3}} \frac{\delta S}{\delta \phi} & =-\left[\ddot{\phi}+3 \frac{\dot{a}}{a} \dot{\phi}+\omega^{2} \phi+6 \xi\left(\frac{\ddot{a}}{a}+\frac{\dot{a}^{2}}{a^{2}}+\frac{K}{a^{2}}\right) \phi\right] \\ & =0\end{aligned}$

$$
\frac{1}{a^{3}} \frac{\delta S}{\delta \varphi}=-\left(\ddot{\varphi}+3 \frac{\dot{a}}{a} \dot{\varphi}+m^{2} \varphi+\frac{1}{2} V_{\varphi}^{\prime}\right)=0 .
$$

It is also easy to verify that the Hamiltonian constraint (6) is conserved for any $\xi$,

$$
\frac{\mathrm{d} H}{\mathrm{~d} \tau}+\dot{a} \frac{\delta S}{\delta a}+\dot{\phi} \frac{\delta S}{\delta \phi}+\dot{\varphi} \frac{\delta S}{\delta \varphi}=0 .
$$

\section{B. Hamiltonian dynamics}

The conjugate momenta are given by

$$
\begin{aligned}
& P_{N}=\frac{\partial \mathcal{L}}{\partial \dot{N}}=0 \\
& P_{a}=\frac{\partial \mathcal{L}}{\partial \dot{a}}=\frac{6 a}{N}\left[\xi a \phi \dot{\phi}-\dot{a}\left(v-\xi \phi^{2}\right)\right] \\
& P_{\phi}=\frac{\partial \mathcal{L}}{\partial \dot{\phi}}=\frac{a^{2}}{N}(a \dot{\phi}+6 \xi \dot{a} \phi) \\
& P_{\varphi}=\frac{\partial \mathcal{L}}{\partial \dot{\varphi}}=\frac{a^{3} \dot{\varphi}}{N}
\end{aligned}
$$

and one can re-express the super-Hamiltonian as

$$
\begin{aligned}
H= & -\frac{\left(a P_{a}-6 \xi \phi P_{\phi}\right)^{2}}{12 a^{3} W_{\xi}(\phi)}+\frac{P_{\phi}^{2}}{2 a^{3}}-3 K a\left(v-\xi \phi^{2}\right) \\
& +\frac{P_{\varphi}^{2}}{2 a^{3}}+\frac{a^{3}}{2}\left(\omega^{2} \phi^{2}+m^{2} \varphi^{2}+V_{\varphi}\right),
\end{aligned}
$$

where we have again set $N=1$ and

$$
W_{\xi}(\phi)=v-\xi(1-6 \xi) \phi^{2} .
$$


Of course, equivalent expressions of the superHamiltonian can be implemented by applying canonical transformations. For example, in order to quantise the system canonically, it is more convenient to use $\bar{H} \equiv W_{\xi}(\phi) H$ (see Ref. [6] for the details), namely

$$
\begin{aligned}
\bar{H}= & -\frac{\left(a P_{a}-6 \xi \phi P_{\phi}\right)^{2}}{12 a^{3}} \\
& +\frac{W_{\xi}(\phi)}{2 a^{3}}\left[P_{\phi}^{2}+P_{\varphi}^{2}-6 K a^{4}\left(v-\xi \phi^{2}\right)\right. \\
& \left.+a^{6}\left(\omega^{2} \phi^{2}+m^{2} \varphi^{2}+V_{\varphi}\right)\right] .
\end{aligned}
$$

\section{SEMICLASSICAL MODEL}

The quantization of the system is realized canonically, i.e. by replacing the classical variables and momenta with the respective operators. The Hamiltonian constraint $\bar{H}=0$ then becomes the WDW equation [12]

$$
\hat{H}|\Psi\rangle=0
$$

A convenient way to treat this equation is to operate the BO factorization [3, 4, 6 ]

$$
\Psi(a, \phi, \varphi)=\psi(a) X(\phi, \varphi ; a)
$$

The operators $\hat{P}_{a}, \hat{P}_{\phi}$ and $\hat{P}_{\varphi}$ are defined as usual,

$$
\hat{P}_{a}=-i \hbar \partial_{a}, \quad \hat{P}_{\phi}=-i \hbar \partial_{\phi}, \quad \hat{P}_{\varphi}=-i \hbar \partial_{\varphi}
$$

and we also define the scalar product

$$
\langle X \mid Y\rangle=\int \mathrm{d} \phi \mathrm{d} \varphi X^{*}(\phi, \varphi ; a) Y(\phi, \varphi ; a) .
$$

It is now convenient to factor out a geometrical phase and redefine the wavefunctions as

$$
\begin{gathered}
\psi \rightarrow \tilde{\psi}=e^{+\frac{i}{\hbar} \int\left\langle X\left|\hat{P}_{a}\right| X\right\rangle \mathrm{d} a} \psi \\
X \rightarrow \tilde{X}=e^{-\frac{i}{\hbar} \int\left\langle X\left|\hat{P}_{a}\right| X\right\rangle \mathrm{d} a} X,
\end{gathered}
$$

from which it follows that $\psi X=\tilde{\psi} \tilde{X}$ and

$$
\left\langle\tilde{X}\left|\hat{P}_{a}\right| \tilde{X}\right\rangle=0
$$

We shall omit tildes from now on.

\section{A. Gravitational equation}

We obtain the equation for the gravitational wavefunction $\psi$ by contracting the WDW equation with $\langle X|$, and using Eq. (20),

$$
\left\langle X\left|\hat{O}(\phi, \psi) \hat{P}_{a}\right| X \psi\right\rangle=\left(\left\langle\hat{O} \hat{P}_{a}\right\rangle+\langle\hat{O}\rangle \hat{P}_{a}\right)|\psi\rangle,
$$

where $\hat{O}$ is any operator and $\langle\hat{O}\rangle \equiv\langle X|\hat{O}| X\rangle$. In details,

$$
\begin{aligned}
& \frac{1}{2}\left\{-\frac{1}{6 a^{3}}\left[a^{2} \hat{P}_{a}^{2}-12 \xi\left\langle\hat{\phi} \hat{P}_{\phi}\right\rangle a \hat{P}_{a}+36 \xi^{2}\left\langle\hat{\phi}^{2} \hat{P}_{\phi}^{2}\right\rangle\right]\right. \\
& +\frac{1}{a^{3}}\left[\left\langle\hat{W}_{\xi} \hat{P}_{\phi}^{2}\right\rangle+\left\langle\hat{W}_{\xi} \hat{P}_{\varphi}^{2}\right\rangle\right]-6 K a\left\langle\hat{W}_{\xi}\left(v-\xi \hat{\phi}^{2}\right)\right\rangle \\
& \left.\quad+a^{3}\left[\omega^{2}\left\langle\hat{W}_{\xi} \hat{\phi}^{2}\right\rangle+m^{2}\left\langle\hat{W}_{\xi} \hat{\varphi}^{2}\right\rangle+\left\langle\hat{W}_{\xi} \hat{V}_{\varphi}\right\rangle\right]\right\}|\psi\rangle \\
& =\frac{1}{2}\left(\frac{\left\langle\hat{P}_{a}^{2}\right\rangle}{6 a}-\frac{2 \xi}{a^{2}}\left\langle\hat{\phi} \hat{P}_{\phi} \hat{P}_{a}\right\rangle\right)|\psi\rangle \equiv \hat{\Delta}^{(g)}|\psi\rangle .
\end{aligned}
$$

Assuming that the effect of $\hat{\Delta}^{(g)}$ is negligible and that $\psi$ is peaked on a classical trajectory $a=a(\tau)$, we can employ the WKB approximation, neglecting terms of order $\hbar^{2}$ or higher,

$$
\psi \simeq \psi_{\mathrm{WKB}}=e^{+\frac{i}{\hbar}\left(S_{0}+\hbar S_{1}\right)}
$$

where $S_{0}$ and $S_{1}$ are implicitly defined by the relations

$$
\begin{aligned}
& \partial_{a} S_{0}=P_{a} \\
& \partial_{a} S_{1}=f(a) \equiv \frac{i}{2} \frac{\partial_{a} P_{a}}{P_{a}-z(a)} \\
& z(a)=\frac{6 \xi}{a}\left\langle\hat{\phi} \hat{P}_{\phi}\right\rangle,
\end{aligned}
$$

whence

$$
\hat{P}_{a} \psi_{\mathrm{WKB}}=\left[P_{a}+\hbar f(a)\right] \psi_{\mathrm{WKB}} .
$$

This allows us to write the semiclassical Hamilton-Jacobi equation for $a=a(\tau)$,

$$
\begin{aligned}
& 3 a\left(\dot{a}^{2}+K \frac{\left\langle\hat{W}_{\xi}\left(v-\xi \hat{\phi}^{2}\right)\right\rangle}{\left\langle\hat{W}_{\xi}\right\rangle^{2}}\right)-\frac{\left\langle\hat{W}_{\xi} \hat{P}_{\phi}^{2}\right\rangle+\left\langle\hat{W}_{\xi} \hat{P}_{\varphi}^{2}\right\rangle}{2 a^{3}\left\langle\hat{W}_{\xi}\right\rangle^{2}} \\
& +\frac{a^{3}}{2\left\langle\hat{W}_{\xi}\right\rangle^{2}}\left(\omega^{2}\left\langle\hat{W}_{\xi} \hat{\phi}^{2}\right\rangle+m^{2}\left\langle\hat{W}_{\xi} \hat{\varphi}^{2}\right\rangle+\left\langle\hat{W}_{\xi} \hat{V}_{\varphi}\right\rangle\right) \\
& =-3 \xi^{2} \frac{\left\langle\hat{\phi}^{2} \hat{P}_{\phi}^{2}\right\rangle-\left\langle\hat{\phi} \hat{P}_{\phi}\right\rangle^{2}}{a^{3}\left\langle\hat{W}_{\xi}\right\rangle^{2}} \equiv \Delta_{\phi} .
\end{aligned}
$$

in which we used the semiclassical expression for $P_{a}$,

$$
P_{a}=6 \frac{\xi}{a}\left\langle\hat{\phi} \hat{P}_{\phi}\right\rangle-6 a \dot{a}\left\langle\hat{W}_{\xi}\right\rangle
$$

Neglecting $\Delta_{\phi}$, approximating the mean values of products of operators with the products of their mean values, replacing $\hat{P}_{\phi}$ and $\hat{P}_{\varphi}$ with their classical expressions, and expanding $\hat{W}_{\xi}$, one can finally recover the semiclassical gravitational equation (6). 


\section{B. Matter equation}

The equation for the evolution of matter states is obtained by computing

$$
\hat{H}|X \psi\rangle-|X\rangle\langle X|\hat{H}| X \psi\rangle=0 \text {. }
$$

Note that the procedure defined by this formula implies that our matter equations will always contain structures of the form $\hat{O}-\langle\hat{O}\rangle$ for relevant operators $\hat{O}$, and this will turn out very important, for example, for the operator ordering [see the discussion after Eq. (54)].

We can introduce the (proper) time according to

$$
\dot{a} \hat{P}_{a}=-i \hbar \dot{a} \frac{\partial}{\partial a} \equiv-i \hbar \frac{\partial}{\partial \tau}
$$

and, using (25), Eq. (28) yields

$$
\left[i \hbar \partial_{\tau}-(\hat{H}-\langle\hat{H}\rangle)\right]|X\rangle=\hat{\Delta}^{(m)}|X\rangle
$$

where

$$
\hat{H}=\hat{H}_{\phi}+\frac{\hat{W}_{\xi}}{\left\langle\hat{W}_{\xi}\right\rangle} \hat{H}_{\varphi}
$$

and

$$
\begin{aligned}
\hat{\Delta}^{(m)}= & -\frac{1}{12 a\left\langle\hat{W}_{\xi}\right\rangle}\left\{\hat{P}_{a}^{2}-\left\langle\hat{P}_{a}^{2}\right\rangle\right. \\
& -12 \frac{\xi}{a}\left[\left(\hat{\phi} \hat{P}_{\phi}-\left\langle\hat{\phi} \hat{P}_{\phi}\right\rangle\right) \hat{P}_{a}-\left\langle\hat{\phi} \hat{P}_{\phi} \hat{P}_{a}\right\rangle\right] \\
& +\frac{i \hbar}{a \dot{a}}\left(\dot{a}+\frac{a \ddot{a}}{\dot{a}}+a \dot{a} \frac{\partial_{a}\left\langle\hat{W}_{\xi}\right\rangle}{\left\langle\hat{W}_{\xi}\right\rangle}\right. \\
& \left.\left.+\xi \frac{\left\langle\hat{\phi} \hat{P}_{\phi}\right\rangle-a \partial_{a}\left\langle\hat{\phi} \hat{P}_{\phi}\right\rangle}{a^{2}\left\langle\hat{W}_{\xi}\right\rangle}\right) \hat{P}_{a}\right\} .
\end{aligned}
$$

The two Hamiltonians are given by

$$
\begin{aligned}
\hat{H}_{\phi}= & \frac{\hat{W}_{\xi}}{2\left\langle\hat{W}_{\xi}\right\rangle}\left[\frac{\hat{P}_{\phi}^{2}}{a^{3}}+\omega^{2} a^{3} \hat{\phi}^{2}-6 K a\left(v-\xi \hat{\phi}^{2}\right)\right] \\
& -6 \xi \frac{\dot{a}}{a} \hat{\phi} \hat{P}_{\phi}+\frac{3 \xi^{2}}{a^{3}\left\langle\hat{W}_{\xi}\right\rangle}\left(2\left\langle\hat{\phi} \hat{P}_{\phi}\right\rangle \hat{\phi} \hat{P}_{\phi}-\hat{\phi}^{2} \hat{P}_{\phi}^{2}\right) \\
& +\frac{i \hbar \xi}{2 a^{3} \dot{a}\left\langle\hat{W}_{\xi}\right\rangle^{2}}\left[\left(\dot{a}+\frac{a \ddot{a}}{\dot{a}}\right)\left\langle\hat{W}_{\xi}\right\rangle+a \dot{a} \partial_{a}\left\langle\hat{W}_{\xi}\right\rangle\right. \\
& \left.+\frac{\xi}{a^{2}}\left(\left\langle\hat{\phi} \hat{P}_{\phi}\right\rangle-a \partial_{a}\left\langle\hat{\phi} \hat{P}_{\phi}\right\rangle\right)\right] \hat{\phi} \hat{P}_{\phi} \\
\hat{H}_{\varphi}= & \frac{1}{2}\left[\frac{\hat{P}_{\varphi}^{2}}{a^{3}}+a^{3}\left(m^{2} \hat{\varphi}^{2}+\hat{V}_{\varphi}\right)\right]
\end{aligned}
$$

Upon neglecting $\hat{\Delta}^{(m)}$ and rescaling $|X\rangle$ as

$$
X \rightarrow \bar{X}=X e^{-\frac{i}{\hbar} \int\langle\hat{H}\rangle \mathrm{d} \tau}
$$

we recover the usual Schrödinger equation for the full matter wavefunction

$$
i \hbar \partial_{\tau}|\bar{X}\rangle=\hat{H}|\bar{X}\rangle
$$

\section{Separating the Schrödinger equation}

The scalar fields $\phi$ and $\varphi$ still appear together in Eq. (36). However, if we write the matter wavefunction as the product of a wavefunction for the inflaton and one for the perturbation,

$$
X(\phi, \varphi ; a)=\chi(\phi ; a) \rho(\varphi ; a)
$$

we can derive two separate Schrödinger equations for the wavefunctions $\chi$ and $\rho$ from Eq. (30).

To find an evolution equation for the inflaton, we contract Eq. (30) with $\langle\chi|$ and obtain

$$
\left[i \hbar \partial_{\tau}-\left(\hat{H}_{\varphi}-\left\langle\rho\left|\hat{H}_{\varphi}\right| \rho\right\rangle\right)\right]|\rho\rangle=\left\langle\chi\left|\hat{\Delta}^{(m)}\right| \chi \rho\right\rangle
$$

where the right hand side is given by

$$
\begin{aligned}
\left\langle\chi\left|\hat{\Delta}^{(m)}\right| \chi \rho\right\rangle= & -\frac{1}{12 a\left\langle\hat{W}_{\xi}\right\rangle}\left[\hat{P}_{a}^{2}-\left\langle\rho\left|\hat{P}_{a}^{2}\right| \rho\right\rangle\right. \\
& +\frac{i \hbar}{a}\left(1+\frac{a \ddot{a}}{\dot{a}^{2}}+a \frac{\partial_{a}\left\langle\hat{W}_{\xi}\right\rangle}{\left\langle\hat{W}_{\xi}\right\rangle}\right. \\
& \left.\left.+\xi \frac{\left\langle\hat{\phi} \hat{P}_{\phi}\right\rangle-a \partial_{a}\left\langle\hat{\phi} \hat{P}_{\phi}\right\rangle}{\dot{a} a^{2}\left\langle\hat{W}_{\xi}\right\rangle}\right) \hat{P}_{a}\right]|\rho\rangle,
\end{aligned}
$$

in which we used

$$
\left(\left\langle\chi\left|\hat{\phi} \hat{P}_{\phi} \hat{P}_{a}\right| \chi\right\rangle-\left\langle\hat{\phi} \hat{P}_{\phi} \hat{P}_{a}\right\rangle\right)|\rho\rangle=0
$$

We will assume that the effect of the quantum fluctuations (39) on the background can be neglected. Hence, by rescaling $\rho$ as usual,

$$
\rho \rightarrow \bar{\rho}=\rho e^{-\frac{i}{\hbar} \int\left\langle\rho\left|\hat{H}_{\varphi}\right| \rho\right\rangle \mathrm{d} \tau}
$$

Eq. (38) becomes a Schrödinger equation for the inflaton in which the field $\phi$ does not enter explicitly. In the WKB approximation for $\rho$ (as well as $a$ ) one can therefore consider solutions to the classical Einstein equations which are not affected by the presence of the perturbation $\phi$ (the particular case of de Sitter inflation will be analysed in the following Section).

We shall likewise obtain the evolution equation for the perturbing field $\phi$. Contracting equation (30) with $\langle\rho|$, we get the following equation for $\chi$,

$$
\begin{aligned}
& {\left[i \hbar \partial_{\tau}-\hat{H}_{\phi}+\left\langle\chi\left|\hat{H}_{\phi}\right| \chi\right\rangle-\left(\frac{\hat{W}_{\xi}}{\left\langle\hat{W}_{\xi}\right\rangle}-1\right)\left\langle\rho\left|\hat{H}_{\varphi}\right| \rho\right\rangle\right]|\chi\rangle} \\
& \quad=\left\langle\rho\left|\hat{\Delta}^{(m)}\right| \rho \chi\right\rangle
\end{aligned}
$$


where

$$
\begin{aligned}
\left\langle\rho\left|\hat{\Delta}^{(m)}\right| \rho \chi\right\rangle= & -\frac{1}{12 a\left\langle\hat{W}_{\xi}\right\rangle}\left\{\hat{P}_{a}^{2}-\left\langle\chi\left|\hat{P}_{a}^{2}\right| \chi\right\rangle\right. \\
& -12 \frac{\xi}{a}\left[\left(\hat{\phi} \hat{P}_{\phi}-\left\langle\hat{\phi} \hat{P}_{\phi}\right\rangle\right) \hat{P}_{a}-\left\langle\hat{\phi} \hat{P}_{\phi} \hat{P}_{a}\right\rangle\right] \\
& +\frac{i \hbar}{a}\left(1+\frac{a \ddot{a}}{\dot{a}^{2}}+a \frac{\partial_{a}\left\langle\hat{W}_{\xi}\right\rangle}{\left\langle\hat{W}_{\xi}\right\rangle}\right. \\
& \left.\left.+\xi \frac{\left\langle\hat{\phi} \hat{P}_{\phi}\right\rangle-a \partial_{a}\left\langle\hat{\phi} \hat{P}_{\phi}\right\rangle}{\dot{a} a^{2}\left\langle\hat{W}_{\xi}\right\rangle}\right) \hat{P}_{a}\right\}|\chi\rangle .
\end{aligned}
$$

It is worth noting that the inflaton $\varphi$ explicitly contributes to the dynamics of $\chi$ whenever the operator $\hat{W}_{\xi}$ does not act trivially on $\chi$. It is only in that case that one obtains

$$
\left(i \hbar \partial_{\tau}-\hat{H}_{\phi}\right)|\bar{\chi}\rangle=0,
$$

after neglecting $\left\langle\rho\left|\hat{\Delta}^{(m)}\right| \rho \chi\right\rangle$ and rescaling

$$
\chi \rightarrow \bar{\chi}=\chi e^{-\frac{i}{\hbar} \int\left\langle\chi\left|\hat{H}_{\phi}\right| \chi\right\rangle \mathrm{d} \tau} .
$$

\section{DE SITTER INFLATION}

Since the minimally coupled case $\xi=0$ on the flat $(K=0)$ de Sitter space-time has already been studied in details in Ref. [3], we shall here apply the general formalism developed so far to the conformal case $\xi=1 / 6$ and $\mu=0$ with

$$
a(\tau)=a_{0} e^{\mathcal{H} \tau},
$$

where $\mathcal{H}$ is the Hubble constant and we set $a(0)=a_{0}$ for the arbitrary value of the scale factor at proper time $\tau=0$ [13]. We also require that the matter mode $k$ lies inside the de Sitter horizon, at least at the initial time $\tau=\tau_{0}$, so that

$$
k>\mathcal{H} a\left(\tau_{0}\right) .
$$

Eq. (43) then simplifies considerably, since $W_{1 / 6}=v$ and

$$
\begin{aligned}
\left\langle\rho\left|\hat{\Delta}^{(m)}\right| \rho \chi\right\rangle= & -\frac{\ell_{\mathrm{P}}^{2}}{12 a \hbar}\left\{\hat{P}_{a}^{2}-\left\langle\chi\left|\hat{P}_{a}^{2}\right| \chi\right\rangle\right. \\
-\frac{2}{a}\left[\left(\hat{\phi} \hat{P}_{\phi}-\left\langle\chi\left|\hat{\phi} \hat{P}_{\phi}\right| \chi\right\rangle-i \hbar\right) \hat{P}_{a}\right. & \left.\left.\quad-\left\langle\chi\left|\hat{\phi} \hat{P}_{\phi} \hat{P}_{a}\right| \chi\right\rangle\right]\right\}|\chi\rangle
\end{aligned}
$$

where $\ell_{\mathrm{P}}=v^{-1 / 2}$ is the Planck length. All these contributions, once evaluated on an invariant eigenstate (see next Subsection), will turn out to be of order $\hbar \ell_{\mathrm{P}}^{2}$.

The Hamiltonian is now given by

$$
\begin{aligned}
\hat{H}_{\phi} & =\frac{1}{2}\left(\frac{\hat{P}_{\phi}^{2}}{a^{3}}+\omega^{2} a^{3} \hat{\phi}^{2}\right)-\mathcal{H} \hat{\phi} \hat{P}_{\phi}+\ell_{\mathrm{P}}^{2} \hat{H}_{p} \\
& =\hat{H}_{0}+\ell_{\mathrm{P}}^{2} \hat{H}_{p}
\end{aligned}
$$

where we have explicitly separated the perturbing Hamiltonian

$$
\hat{H}_{p}=-\frac{\hat{\phi}^{2} \hat{P}_{\phi}^{2}}{12 a^{3} \hbar}+\frac{\left\langle\hat{\phi} \hat{P}_{\phi}\right\rangle \hat{\phi} \hat{P}_{\phi}}{6 a^{3} \hbar}+\frac{i \hat{\phi} \hat{P}_{\phi}}{6 a^{3}} .
$$

A final simplification can be obtained by re-phasing $\chi$ according to

$$
\chi \rightarrow \chi_{s}=\chi e^{-\frac{i}{\hbar} \int\left\langle\chi\left|\hat{H}_{0}\right| \chi\right\rangle \mathrm{d} \tau}
$$

which yields

$$
\left(i \hbar \partial_{\tau}-\hat{H}_{0}\right)\left|\chi_{s}\right\rangle=\hat{\Delta}_{s}\left|\chi_{s}\right\rangle
$$

where

$$
\begin{aligned}
\hat{\Delta}_{s}= & \frac{\ell_{\mathrm{P}}^{2}}{6 \hbar a^{3}}\left\{\frac{1}{2}\left(\left\langle\hat{\phi}^{2} \hat{P}_{\phi}^{2}\right\rangle_{s}-\hat{\phi}^{2} \hat{P}_{\phi}^{2}\right)\right. \\
& +\left(\frac{\left\langle\hat{H}_{0}\right\rangle_{s}}{\mathcal{H}}+\left\langle\hat{\phi} \hat{P}_{\phi}\right\rangle_{s}+i \hbar\right)\left(\hat{\phi} \hat{P}_{\phi}-\left\langle\hat{\phi} \hat{P}_{\phi}\right\rangle_{s}\right) \\
& -\frac{i \hbar}{\mathcal{H}}\left(\hat{\phi} \hat{P}_{\phi} \partial_{\tau}-\left\langle\hat{\phi} \hat{P}_{\phi} \partial_{\tau}\right\rangle_{s}\right)+\frac{\hbar^{2}}{2 \mathcal{H}^{2}}\left(\partial_{\tau}^{2}-\left\langle\partial_{\tau}^{2}\right\rangle_{s}\right) \\
& +\frac{i \hbar}{\mathcal{H}}\left[\frac{\left\langle\hat{H}_{0}\right\rangle_{s}}{\mathcal{H}}+\frac{i}{2} \hbar\right]\left(\partial_{\tau}-\left\langle\partial_{\tau}\right\rangle_{s}\right) \\
& \left.+\frac{1}{\mathcal{H}}\left[\left\langle\hat{\phi} \hat{P}_{\phi}\right\rangle_{s}+i \hbar\right]\left(i \hbar \partial_{\tau}-\left\langle\hat{H}_{0}\right\rangle_{s}\right)\right\}
\end{aligned}
$$

with $\langle\hat{O}\rangle_{s}=\left\langle\chi_{s}|\hat{O}| \chi_{s}\right\rangle$ for any operator $\hat{O}$.

So far no particular operator ordering was chosen for $\hat{\phi}$ and $\hat{P}_{\phi}$. We shall now choose the Weyl ordering by symmetrising $\hat{\Delta}_{s}$ in $\hat{\phi}$ and $\hat{P}_{\phi}[9]$ according to

$$
\begin{aligned}
& 2 \hat{\phi} \hat{P}_{\phi} \rightarrow \hat{\phi} \hat{P}_{\phi}+\hat{P}_{\phi} \hat{\phi}=2 \hat{\phi} \hat{P}_{\phi}-i \hbar \\
& \hat{\phi}^{2} \hat{P}_{\phi}^{2} \rightarrow \hat{\phi}^{2} \hat{P}_{\phi}^{2}-2 i \hbar \hat{\phi} \hat{P}_{\phi}-\hbar^{2} / 2 .
\end{aligned}
$$

Any other ordering would eventually result in different cnumber terms (proportional to $\hbar^{2}$ ) which do not appear in $\hat{\Delta}_{s}$ due to the general form $\hat{\Delta}_{s} \sim \hat{O}-\langle\hat{O}\rangle_{s}$ [as we noted after Eq. (28)]. Substituting in Eq. (52), after some algebra, one obtains

$$
\left(1-\frac{\ell_{\mathrm{P}}^{2} \Sigma}{6 \mathcal{H} a^{3}}\right)\left(i \hbar \partial_{\tau}-\hat{H}_{0}\right)\left|\chi_{s}\right\rangle=\frac{\ell_{\mathrm{P}}^{2} \hat{\Delta}}{6 \mathcal{H} a^{3}}\left|\chi_{s}\right\rangle
$$

where

$$
\Sigma=\frac{i}{2}+\frac{\left\langle\hat{\phi} \hat{P}_{\phi}\right\rangle_{s}}{\hbar}
$$


and

$$
\begin{aligned}
\hat{\Delta}= & \frac{\mathcal{H}}{2 \hbar}\left(\left\langle\hat{\phi}^{2} \hat{P}_{\phi}^{2}\right\rangle_{s}-\hat{\phi}^{2} \hat{P}_{\phi}^{2}\right) \\
& +\frac{\mathcal{H}}{\hbar}\left(\frac{\left\langle\hat{H}_{0}\right\rangle_{s}}{\mathcal{H}}+\left\langle\hat{\phi} \hat{P}_{\phi}\right\rangle_{s}+\frac{3}{2} i \hbar\right)\left(\hat{\phi} \hat{P}_{\phi}-\left\langle\hat{\phi} \hat{P}_{\phi}\right\rangle_{s}\right) \\
& -i\left(\hat{\phi} \hat{P}_{\phi} \partial_{\tau}-\left\langle\hat{\phi} \hat{P}_{\phi} \partial_{\tau}\right\rangle_{s}\right)+\frac{\hbar}{2 \mathcal{H}}\left(\partial_{\tau}^{2}-\left\langle\partial_{\tau}^{2}\right\rangle_{s}\right) \\
& +i\left(\frac{\left\langle\hat{H}_{0}\right\rangle_{s}}{\mathcal{H}}+i \hbar\right)\left(\partial_{\tau}-\left\langle\partial_{\tau}\right\rangle_{s}\right) \\
& +\left(\frac{\left\langle\hat{\phi} \hat{P}_{\phi}\right\rangle_{s}}{\hbar}+\frac{i}{2}\right)\left(\hat{H}_{0}-\left\langle\hat{H}_{0}\right\rangle_{s}\right) .
\end{aligned}
$$

These are the expressions which we shall estimate in the following.

\section{A. Perturbative analysis}

We now proceed to solve the matter equation (55) perturbatively (in $\ell_{\mathrm{P}}$ ). As can be seen by looking at Eq. (55) and (57) [see also the discussion following Eq. (62)], the terms denoted by $\Sigma$ and $\Delta$ represent a perturbation to the usual Schrödinger equation only when

$$
\epsilon=\frac{\ell_{\mathrm{P}}^{2}}{6 \mathcal{H} a^{3}} \ll 1,
$$

so, strictly speaking, it is only for (relatively) late times such that [14]

$$
a(\tau) \gg\left(\frac{\ell_{\mathrm{P}}^{2}}{6 \mathcal{H}}\right)^{1 / 3} \sim \ell_{\mathrm{P}},
$$

that the perturbative treatment makes sense. In what follows, we will then assume that this relation holds and neglect terms proportional to $\epsilon^{2}$, so that

$$
\frac{\epsilon \hat{\Delta}}{1-\epsilon \Sigma} \simeq \epsilon \hat{\Delta} .
$$

However, we shall also discuss what might happen outside of this regime.

To zero order in $\epsilon$, the matter equation is just [15]

$$
\left(i \hbar \partial_{\tau}-\hat{H}_{0}\right)\left|\chi_{s}\right\rangle=0
$$

with an Hamiltonian of the form

$$
\begin{aligned}
\hat{H}_{0} & =\frac{1}{2}\left(\frac{\hat{P}_{\phi}^{2}}{a^{3}}+\omega^{2} a^{3} \hat{\phi}^{2}\right)-\frac{\mathcal{H}}{2}\left(\hat{\phi} \hat{P}_{\phi}+\hat{P}_{\phi} \hat{\phi}\right) \\
& =\frac{\hbar k}{a}\left(\hat{b}^{\dagger} \hat{b}+\frac{1}{2}\right)-\frac{i}{2} \hbar \mathcal{H}\left[\left(\hat{b}^{\dagger}\right)^{2}-\hat{b}^{2}\right],
\end{aligned}
$$

in which the invariant operators $\hat{b}$ and $\hat{b}^{\dagger}$ are defined in Appendix A. Note that the squeezing term containing $\left(\hat{b}^{\dagger}\right)^{2}$ and $\hat{b}^{2}$ vanishes for vanishing $\mathcal{H}$, although this limit is critical for our treatment in that the definition of time (29) loses its meaning for $\dot{a} \rightarrow 0$ [16]. In particular, on assuming that matter modes are generated in a Bunch-Davies vacuum [tantamount to our condition (A15)] and using Eqs. (A32)-(A36), the effect of the perturbation $\hat{\Delta}$ given in Eq. (57) on invariant eigenstates $\left|\chi_{s}\right\rangle=|n\rangle$ can be easily estimated as

$$
\epsilon \hat{\Delta} \simeq \epsilon \frac{\hbar \mathcal{H}}{4}\left[\left(\hat{b}^{\dagger}\right)^{2}-\hat{b}^{2}\right]=\frac{\ell_{\mathrm{P}}^{2} \hbar}{24 a^{3}}\left[\left(\hat{b}^{\dagger}\right)^{2}-\hat{b}^{2}\right] .
$$

Even if the regime $\epsilon \gtrsim 1$ is clearly non-perturbative, one could try to examine in a qualitative way what happens assuming that Eqs. (A32)-(A36) still hold approximately. One then finds

$$
\frac{\epsilon \hat{\Delta}}{1-\epsilon \Sigma} \simeq \frac{1+i \epsilon}{1+\epsilon^{2}} \epsilon \frac{\hbar \mathcal{H}}{4}\left[\left(\hat{b}^{\dagger}\right)^{2}-\hat{b}^{2}\right] .
$$

For $\epsilon \sim 1$, this expression then yields

$$
\frac{\epsilon \hat{\Delta}}{1-\epsilon \Sigma} \simeq \frac{1+i}{8} \hbar \mathcal{H}\left[\left(\hat{b}^{\dagger}\right)^{2}-\hat{b}^{2}\right]
$$

and, for $\epsilon \gg 1$,

$$
\frac{\epsilon \hat{\Delta}}{1-\epsilon \Sigma} \simeq \frac{i}{4} \hbar \mathcal{H}\left[\left(\hat{b}^{\dagger}\right)^{2}-\hat{b}^{2}\right]
$$

It is remarkable that, in all regimes, the correction amounts to a mere renormalization of the coefficient of the squeezing term independent of $k$, so that the effective Schrödinger equation can be written as

$$
\left(i \hbar \partial_{\tau}-\hat{H}_{\text {new }}\right)\left|\chi_{s}\right\rangle=0
$$

where

$$
\hat{H}_{\text {new }}=\frac{\hbar k}{a}\left(\hat{b}^{\dagger} \hat{b}+\frac{1}{2}\right)-\frac{i}{2} \beta \hbar \mathcal{H}\left[\left(\hat{b}^{\dagger}\right)^{2}-\hat{b}^{2}\right],
$$

and

$$
\beta= \begin{cases}1+\frac{i \ell_{\mathrm{P}}^{2}}{12 \mathcal{H} a^{3}} & \text { for } \quad \epsilon \ll 1 \\ \frac{3+i}{4} & \text { for } \quad \epsilon \sim 1 \\ \frac{1}{2} & \text { for } \epsilon \gg 1\end{cases}
$$

Except for the case $\epsilon \gg 1$, the operator $\hat{H}_{\text {new }}$ is however not Hermitian, despite the fact that the approach followed ensures that the evolution of the system remains unitary [1]. Note also that the squeezing term remains negligible for large momenta, that is $a \mathcal{H} / k \ll 1$. 


\section{B. Late time dispersion relation}

The case $\epsilon \ll 1$ is similar to $\ell_{\mathrm{P}}^{2} \mathcal{H}^{2}=\delta^{2} \ll 1$ in Ref. [3] . One can therefore employ the same kind of perturbative expansion,

$$
\left|\chi_{s}\right\rangle=\left|n_{s}\right\rangle \simeq\left(\hat{1}+\delta^{2} \hat{R}_{n}\right)|n\rangle
$$

where $\hat{R}_{n}$ must then satisfy

$$
\left(i \hbar \partial_{\tau} \hat{R}_{n}-\left[\hat{H}_{0}, \hat{R}_{n}\right]\right)|n\rangle=\frac{\epsilon}{\delta^{2}} \hat{\Delta}|n\rangle .
$$

This implies that

$$
\hat{R}_{n}=r \hat{b}^{2}+r^{*}\left(\hat{b}^{\dagger}\right)^{2}
$$

where

$$
i a \dot{r}+2 k r=-\frac{1}{24 \mathcal{H}^{2} a^{2}},
$$

whose solution [assuming $r(\tau \rightarrow \infty)=0$ ] is given by

$$
r(\tau)=\frac{1}{96 k^{3}}\left[1+\frac{2 i k}{\mathcal{H} a}\left(1-\frac{i k}{\mathcal{H} a}\right)-e^{-\frac{2 i k}{\mathcal{H} a}}\right]
$$

from which it is again clear that $\hat{R}_{n}$ is not anti-Hermitian [ $a=a(\tau)$ is given in Eq. (46)].

In order to determine a (modified) dispersion relation for the mode $k$, we first need to determine an effective Hermitian Hamiltonian $\hat{H}_{\text {eff }}$ so that the states $\left|\bar{\chi}_{s}\right\rangle$ evolved by it produce the same expectation values for any observables $\hat{X}$ as those given by $\left|\chi_{s}\right\rangle$ in Eq. (70),

$$
\left\langle\bar{\chi}_{s}|\hat{X}| \bar{\chi}_{s}\right\rangle=\left\langle\chi_{s}|\hat{X}| \chi_{s}\right\rangle
$$

To first order in $\epsilon$, we can write

$$
\hat{H}_{\mathrm{eff}}=i \hbar\left(\partial_{\tau} \hat{U}\right) \hat{U}^{-1}
$$

where the complete propagator $\hat{U}$ is given by

$$
\hat{U} \simeq\left(\hat{1}+i \delta^{2} \hat{H}_{n}\right) \hat{U}_{0}
$$

with $\hat{U}_{0}$ the propagator for the Schrödinger equation (61), so that

$$
\left|\bar{\chi}_{s}\right\rangle=\left|\bar{n}_{s}\right\rangle=\hat{U}|n\rangle \simeq\left(\hat{1}+i \delta^{2} \hat{H}_{n}\right)|n\rangle .
$$

After some algebra, one finds

$$
\hat{H}_{\mathrm{eff}} \simeq \hat{H}_{0}-\delta^{2}\left(\hbar \partial_{\tau} \hat{H}_{n}+i\left[\hat{H}_{0}, \hat{H}_{n}\right]\right) \text {. }
$$

The Hermitian operator $\hat{H}_{n}$ can be finally determined by imposing the condition (75) for $\hat{X}=\hat{\phi}^{2}, \hat{P}_{\phi}^{2}$ and $\left\{\hat{\phi}, \hat{P}_{\phi}\right\}$, which yields

$$
\hat{H}_{n}=i \frac{n^{2}+n+1}{2 n+1}\left[r \hat{b}^{2}-r^{*}\left(\hat{b}^{\dagger}\right)^{2}\right],
$$

where $r=r(\tau)$ is the same solution to Eq. (73). Putting all the pieces together, the effective Hamiltonian is then given by

$$
\begin{aligned}
\hat{H}_{\mathrm{eff}} & \simeq \hat{H}_{0}+\frac{\ell_{\mathrm{P}}^{2} \hbar}{24 a^{3}}\left[\left(\hat{b}^{\dagger}\right)^{2}+\hat{b}^{2}\right] \\
& =\frac{1}{2}\left(\frac{\hat{P}_{\phi}^{2}}{\mu}+\mu \omega_{\mathrm{eff}}^{2} \hat{\phi}^{2}\right)+\gamma\left(\hat{\phi} \hat{P}_{\phi}+\hat{P}_{\phi} \hat{\phi}\right),
\end{aligned}
$$

with effective mass and frequency

$$
\begin{aligned}
\mu(\xi=1 / 6) & \simeq a^{3}\left(1+\frac{\ell_{\mathrm{P}}^{2}}{12 k a^{2}}\right) \\
\omega_{\mathrm{eff}}(\xi=1 / 6) & \simeq \omega
\end{aligned}
$$

and

$$
\gamma(\xi=1 / 6)=-\frac{\mathcal{H}}{2} .
$$

To make the comparison easier, we report here the results for the minimally coupled case [17],

$$
\begin{aligned}
\mu(\xi=0) & \simeq a^{3}\left(1+\frac{3 \ell_{\mathrm{P}}^{2}}{k a^{2}}\right) \\
\omega_{\mathrm{eff}}(\xi=0) & \simeq \omega\left(1+\frac{3 \ell_{\mathrm{P}}^{2} \mathcal{H}^{2}}{4 k^{3}}\right) \\
\gamma(\xi=0) & \simeq \frac{3 \ell_{\mathrm{P}}^{2} \mathcal{H}}{4 k a^{2}} .
\end{aligned}
$$

The most evident difference is therefore that the effective frequency $\omega_{\text {eff }}(\xi=1 / 6) \simeq \omega$ to first order in $\ell_{\mathrm{P}}^{2}$. The effective mass is changed in both cases by terms proportional to $1 / k$, which can be interpreted as a different "effective" cosmological scale factor $a_{\text {eff }}=\mu^{1 / 3}(k)$ for each matter mode. We remark that matter modes are generated inside the Hubble horizon, so that no infrared divergence occurs in the above expressions by virtue of Eq. (47).

\section{CONCLUSIONS}

We have analysed the dynamics of a scalar field generically coupled to gravity driven by an inflaton following the approach of Refs. [4, 6]. After showing under which conditions one can treat the cosmological scale factor and the inflaton as (coupled) classical degrees of freedom, we specialized to massless conformally coupled matter in de Sitter inflation.

The main conclusion is that, at least in the perturbative regime $\epsilon \ll 1$ corresponding to a relatively large cosmological scale factor (compared to the Planck length), quantum gravitational corrections in the Schrödinger equation are negligible, since one can introduce an effective Hamiltonian (81) with the same effective frequency and squeezing term (to order $\ell_{\mathrm{P}}^{2}$ ) that appear in the usual equation (61) obtained from quantum field theory on that 
classical background. This result can be viewed as an extension to order $\ell_{\mathrm{P}}^{2}$ of the well-known fact that conformally coupled scalar fields evolve freely on a cosmological background and is strikingly different from what we obtained for a minimally coupled scalar field in Ref. [3], for which both the effective frequency and squeezing term instead contain corrections starting at order $\ell_{\mathrm{P}}^{2}$.

\section{APPENDIX A: MATTER STATES FOR GENERAL COUPLING}

The unperturbed Eq. (61) can be solved for a general value of $\xi$ provided

$$
\frac{\hat{W}_{\xi}}{\left\langle\hat{W}_{\xi}\right\rangle} \simeq 1
$$

so that the Hamiltonian $\hat{H}_{0}$ can be expressed as

$$
\hat{H}_{0}=\hbar \omega_{\xi}\left(\hat{a}_{\xi}^{\dagger} \hat{a}_{\xi}+\frac{1}{2}\right)
$$

where

$$
\omega_{\xi}^{2}=\omega^{2}+6 \xi\left(\frac{K}{a^{2}}-6 \xi \frac{\dot{a}^{2}}{a^{2}}\right)
$$

and $\left[\hat{a}_{\xi}, \hat{a}_{\xi}^{\dagger}\right]=1$ with

$$
\hat{a}_{\xi}=\sqrt{\frac{a^{3} \omega_{\xi}}{2 \hbar}}\left[\hat{\phi}+\frac{i}{\omega_{\xi}}\left(\frac{\hat{P}_{\phi}}{a^{3}}-6 \xi \frac{\dot{a}}{a} \hat{\phi}\right)\right] .
$$

For this Hamiltonian, the relevant invariant operators $\left[3,[6,10]\right.$ satisfying $\left[\hat{b}_{\xi}, \hat{b}_{\xi}^{\dagger}\right]=1$ are given by

$$
\left.\hat{b}_{\xi}=i \sqrt{\frac{a^{3} \sigma^{2}}{2 \hbar}}\left\{\frac{\hat{P}_{\phi}}{a^{3}}-\left[\frac{i}{\sigma^{2}}+\frac{\dot{\sigma}}{\sigma}+6 \frac{\dot{a}}{a}\left(\xi-\frac{1}{4}\right)\right] \hat{\phi}\right\} \mathrm{A}, 5\right)
$$

and its Hermitian conjugate. The function $\sigma=\sigma(\tau)$ must be a solution to the Pinney equation

$$
\ddot{\sigma}+\Omega^{2} \sigma=\frac{1}{\sigma^{3}},
$$

with

$$
\Omega^{2}=\omega_{\xi}^{2}+\left(12 \xi-\frac{3}{4}\right) \frac{\dot{a}^{2}}{a^{2}}+\left(6 \xi-\frac{3}{2}\right) \frac{\ddot{a}}{a} .
$$

The eigenstates of the invariant number operator,

$$
\hat{n}_{b}|n\rangle=\hat{b}_{\xi}^{\dagger} \hat{b}_{\xi}|n\rangle=n|n\rangle,
$$

then provide us with the exact solutions we are looking for, namely

$$
|n\rangle=\frac{e^{-i n \Theta(\tau)}}{\sqrt{n !}}\left(\hat{b}_{\xi}^{\dagger}\right)^{n}|0\rangle,
$$

where $\hat{b}_{\xi}|0\rangle=0$ and

$$
\Theta=\int^{\tau} \frac{\mathrm{d} t}{\sigma^{2}(t)} .
$$

For a de Sitter space-time, with $\dot{a}^{2} / a^{2}=\ddot{a} / a=\mathcal{H}^{2}$, we then have

$$
\Omega^{2}=A e^{-2 \mathcal{H} \tau}-B
$$

where

$$
A=k^{2}+6 \xi K, \quad B=36(\xi-1 / 4)^{2} \mathcal{H}^{2}-\mu^{2} .
$$

The general solution to Eq. (A6) can be written as

$$
\sigma=\frac{1}{w} \sqrt{c_{1} h_{1}^{2}+c_{2} h_{2}^{2}+2 \sqrt{c_{1} c_{2}-w^{2}} h_{1} h_{2}},
$$

where $h_{1}$ and $h_{2}$ must be any two linear independent solutions to the auxiliary homogeneous equation

$$
\ddot{\sigma}+\Omega^{2} \sigma=0,
$$

$w=\dot{h}_{1} h_{2}-h_{1} \dot{h}_{2}$ is their Wronskian and $c_{1}, c_{2}$ are coefficients to be fixed by appropriate initial conditions at $\tau=\tau_{0}$. For example, a common requirement is that the invariant number operator and the Hamiltonian are proportional at $\tau=\tau_{0}=-\infty[\underline{3},[\underline{6}]$

$$
\hat{n}_{b}(\tau=-\infty) \propto \hat{H}_{0}(\tau=-\infty) .
$$

This amounts to requiring that

$$
\hat{a}_{\xi}^{\dagger} \hat{a}_{\xi} \propto \hat{b}_{\xi}^{\dagger} \hat{b}_{\xi}, \quad \text { at } \tau=-\infty .
$$

It can be shown that this uniquely implies that

$$
\hat{a}_{\xi} \propto \hat{b}_{\xi}, \quad \hat{a}_{\xi}^{\dagger} \propto \hat{b}_{\xi}^{\dagger}, \quad \text { at } \tau=-\infty,
$$

and from these relations one finds the appropriate initial conditions for $\sigma$ as

$$
\begin{aligned}
& \sigma\left(\tau_{0}=-\infty\right)=\frac{1}{\sqrt{\omega_{\xi}(-\infty)}} \\
& \dot{\sigma}\left(\tau_{0}=-\infty\right)=\frac{3}{2} \mathcal{H} \sigma(-\infty)=\frac{3 \mathcal{H}}{2 \sqrt{\omega_{\xi}(-\infty)}} .
\end{aligned}
$$

It also follows that $|0\rangle$ is then the usual Bunch-Davies vacuum of minimum energy at $\tau=\tau_{0}$.

A convenient pair of solutions $h_{1}$ and $h_{2}$ is given in terms of the Hankel functions

$$
\begin{aligned}
& h_{1}(\tau ; \nu)=i H_{\nu}^{(1)}(z)=i J_{\nu}(z)-N_{\nu}(z) \\
& h_{2}(\tau ; \nu)=i H_{\nu}^{(2)}(z)=i J_{\nu}(z)+N_{\nu}(z),
\end{aligned}
$$

where $H, J$ and $N$ are the Hankel, Bessel and Neumann functions respectively,

$$
\nu=\frac{\sqrt{B}}{\mathcal{H}}, \quad z=\frac{\sqrt{A}}{\mathcal{H}} e^{-\mathcal{H} t},
$$


and $w=i 4 \mathcal{H} / \pi[18]$.

We can now use the large $z$ expressions [19] of the functions $h_{1}$ and $h_{2}$,

$$
\begin{array}{r}
h_{1} \simeq i e^{\frac{\mathcal{H} \tau}{2}} \sqrt{\frac{\mathcal{H}}{\sqrt{A}}} \sqrt{\frac{2}{\pi}}\left[\cos \left(\frac{\mathcal{H}}{\sqrt{A}} e^{-\mathcal{H} \tau}-\frac{\pi}{4}-\frac{\sqrt{B} \pi}{2 \mathcal{H}}\right)\right. \\
\left.+i \sin \left(\frac{\mathcal{H}}{\sqrt{A}} e^{-\mathcal{H} \tau}-\frac{\pi}{4}-\frac{\sqrt{B} \pi}{2 \mathcal{H}}\right)\right] \\
=\sqrt{\frac{\mathcal{H}}{\sqrt{\pi A}}}(i-1) e^{-i\left(\frac{\mathcal{H}}{\sqrt{A}} e^{-\mathcal{H} \tau}-\frac{\sqrt{B} \pi}{2 \mathcal{H}}\right) e^{\frac{\mathcal{H} \tau}{2}}} \quad(\mathrm{~A} 21) \\
h_{2} \simeq i e^{\frac{\mathcal{H} \tau}{2}} \sqrt{\frac{\mathcal{H}}{\sqrt{A}}} \sqrt{\frac{2}{\pi}}\left[\cos \left(\frac{\mathcal{H}}{\sqrt{A}} e^{-\mathcal{H} \tau}-\frac{\pi}{4}-\frac{\sqrt{B} \pi}{2 \mathcal{H}}\right)\right. \\
=\sqrt{\frac{\mathcal{H}}{\sqrt{\pi A}}}(1+i) e^{i\left(\frac{\mathcal{H}}{\sqrt{A}} e^{-\mathcal{H} t}-\frac{\sqrt{\mathcal{B}} \pi}{2 \mathcal{H}}\right) e^{\frac{\mathcal{H} t}{2}} .} \quad \text { (A22) }
\end{array}
$$

Then, on considering that

$$
\omega_{\xi} \simeq \sqrt{A} e^{-\mathcal{H} \tau}, \quad \text { for } \tau \rightarrow-\infty,
$$

and imposing the initial conditions (A18) on Eq. (A6), one finds that $c_{1}$ and $c_{2}$ as function of the initial time $\tau_{0}$ must decay exponentially for $\tau_{0} \rightarrow-\infty$,

$$
\begin{aligned}
& c_{1}=\omega_{\xi} h_{2}^{2}+\frac{\left(2 \dot{h}_{2}-3 \mathcal{H} h_{2}\right)^{2}}{4 \omega_{\xi}} \\
& \simeq-\frac{6 \mathcal{H}^{2}}{A \pi} e^{\mathcal{H} \tau_{0}+i\left(\frac{2 \mathcal{H}}{\sqrt{A}} e^{-\mathcal{H} \tau_{0}}-\frac{\sqrt{B}}{\mathcal{H}} \pi\right)}\left(\sqrt{A}-\frac{3}{4} i \mathcal{H} e^{\mathcal{H} \tau_{0}}\right) \\
& c_{2}=\omega_{\xi} h_{1}^{2}+\frac{\left(2 \dot{h}_{1}-3 \mathcal{H} h_{1}\right)^{2}}{4 \omega_{\xi}} \\
& \simeq-\frac{6 \mathcal{H}^{2}}{A \pi} e^{\mathcal{H} \tau_{0}+i\left(\frac{\sqrt{B}}{\mathcal{H}} \pi-\frac{2 \mathcal{H}}{\sqrt{A}} e^{-\mathcal{H} \tau_{0}}\right)}\left(\sqrt{A}+\frac{3}{4} i \mathcal{H} e^{\mathcal{H} \tau_{0}}\right),
\end{aligned}
$$

and we can set

$$
c_{1}=c_{2}=0 \text {. }
$$

Finally, the solution to the Pinney equation (A6) fulfilling these initial conditions is

$$
\sigma=\sqrt{\frac{\pi}{2 \mathcal{H}}} \sqrt{J_{\frac{\sqrt{B}}{\mathcal{H}}}^{2}\left(\frac{\sqrt{A}}{\mathcal{H}} e^{-\mathcal{H} \tau}\right)+N_{\frac{\sqrt{B}}{\mathcal{H}}}^{2}\left(\frac{\sqrt{A}}{\mathcal{H}} e^{-\mathcal{H} \tau}\right)} .
$$

For a conformally coupled $(\xi=1 / 6)$ massless $(\mu=0)$ scalar field in spatially flat $(K=0)$ de Sitter, one has $W_{1 / 6}=v$ [so that Eq. (A1) hold] and

$$
\omega_{1 / 6}^{2}=\omega^{2}\left[1-\left(\frac{\mathcal{H} a}{k}\right)^{2}\right],
$$

which is real by virtue of the condition (47). The solution to the Pinney equation (A6) then turns out to be very simply

$$
\sigma(\tau)=\frac{1}{\sqrt{\omega}}=\sqrt{\frac{a}{k}} .
$$

The invariant annihilation operator (A5) becomes

$$
\hat{b}=\frac{1}{\sqrt{2 \hbar}}\left(\sqrt{k} a \hat{\phi}+i \frac{\hat{P}_{\phi}}{\sqrt{k} a}\right),
$$

from which

$$
\begin{aligned}
& \hat{\phi}=\sqrt{\frac{\hbar}{2 k a^{2}}}\left(\hat{b}^{\dagger}+\hat{b}\right) \\
& \hat{P}_{\phi}=i \sqrt{\frac{\hbar k a^{2}}{2}}\left(\hat{b}^{\dagger}-\hat{b}\right) .
\end{aligned}
$$

We also obtain the following useful relations

$$
\left(\hat{\phi} \hat{P}_{\phi}-\left\langle\hat{\phi} \hat{P}_{\phi}\right\rangle\right)|n\rangle=\frac{i}{2} \hbar\left[\left(\hat{b}^{\dagger}\right)^{2}-\hat{b}^{2}\right]|n\rangle
$$

$$
\begin{aligned}
& \left(\left\langle\hat{\phi}^{2} \hat{P}_{\phi}^{2}\right\rangle-\hat{\phi}^{2} \hat{P}_{\phi}^{2}\right)|n\rangle \\
& =\frac{\hbar^{2}}{4}\left[\hat{b}^{4}+\left(\hat{b}^{\dagger}\right)^{4}-4 \hat{b}^{2}+4\left(\hat{b}^{\dagger}\right)^{2}\right]|n\rangle
\end{aligned}
$$

$$
\left(\hat{H}_{0}-\left\langle\hat{H}_{0}\right\rangle\right)|n\rangle=\frac{i}{2} \hbar \mathcal{H}\left(\hat{b}^{2}-\left(\hat{b}^{\dagger}\right)^{2}\right)|n\rangle
$$

$$
\begin{aligned}
& \left(\hat{\phi} \hat{P}_{\phi} \partial_{\tau}-\left\langle\hat{\phi} \hat{P}_{\phi} \partial_{\tau}\right\rangle\right)|n\rangle=\frac{i}{4} \hbar \mathcal{H}\left\{\hat{b}^{2}-\left(\hat{b}^{\dagger}\right)^{2}\right. \\
& \left.-\left(\hat{b}^{\dagger}\right)^{4}-\hat{b}^{4}-\frac{i k}{a \mathcal{H}}(2 n+1)\left[\left(\hat{b}^{\dagger}\right)^{2}-\hat{b}^{2}\right]\right\}|n\rangle
\end{aligned}
$$

$$
\begin{aligned}
& \left(\partial_{\tau}^{2}-\left\langle\partial_{\tau}^{2}\right\rangle\right)|n\rangle=-i \frac{k \mathcal{H}}{2 a}\left[(2 n+1)\left(\hat{b}^{2}-\left(\hat{b}^{\dagger}\right)^{2}\right)\right. \\
& \left.+i \frac{\mathcal{H} a}{2 k}\left(\hat{b}^{4}+\left(\hat{b}^{\dagger}\right)^{4}\right)\right]|n\rangle
\end{aligned}
$$

Using Eq. (A29), one can also write the coefficients of the Bogoliubov transformation,

$$
\begin{aligned}
& \hat{b}=\zeta^{*} \hat{a}+\eta \hat{a}^{\dagger} \\
& \hat{b}^{\dagger}=\zeta \hat{a}^{\dagger}+\eta^{*} \hat{a},
\end{aligned}
$$

as

$$
\begin{aligned}
& \eta=\frac{1+i x-\sqrt{1-x^{2}}}{2\left(1-x^{2}\right)^{1 / 4}} \\
& \zeta=\frac{1-i x+\sqrt{1-x^{2}}}{2\left(1-x^{2}\right)^{1 / 4}},
\end{aligned}
$$


where $x=\mathcal{H} a / k$.

[1] A.R. Liddle and D.H. Lyth, Cosmological inflation and large-scale structure (Cambridge University Press, Cambridge, England, 2000).

[2] J. Martin and R.H. Brandenberger, Phys. Rev. D 63, 123501 (2001); U.H. Danielsson, Phys. Rev. D 66, 023511 (2002); V. Bozza, M. Giovannini and G. Veneziano, JCAP 0305, 001 (2003); A.A. Starobinsky, JETP Lett. 73, 371 (2001); R.H. Brandenberger and J. Martin, Phys. Rev. D 71 (2005) 023504; J. Martin and C. Ringeval, JCAP 0608, 009 (2006), JCAP 0501, 007 (2005), Phys. Rev. D 69, 083515 (2004); H. Collins and R. Holman, Phys. Rev. D 74, 045009 (2006), Phys. Rev. D 71, 085009 (2005); B. Greene, M. Parikh and J.P. van der Schaar, JHEP 0604, 057 (2006); A. Ashoorioon, J. L. Hovdebo and R. B. Mann, Nucl. Phys. B 727, 63 (2005); S. Hossenfelder, Mod. Phys. Lett. A 19, 2727 (2004); L. Sriramkumar and T. Padmanabhan, Phys. Rev. D 71, 103512 (2005); J. de Boer, V. Jejjala and D. Minic, Phys. Rev. D 71, 044013 (2005); G. Calcagni, Phys. Rev. D 70, 103525 (2004); S. Shankaranarayanan and L. Sriramkumar, Phys. Rev. D 70, 123520 (2004); R.G. Cai, Phys. Lett. B 593, 1 (2004); M. Porrati, Phys. Lett. B 596 , 306 (2004); M. Fukuma, Y. Kono and A. Miwa, Nucl. Phys. B 703, 293 (2004); K. Ke, Int. J. Mod. Phys. A 20, 4331 (2005); S. Hannestad, JCAP 0404, 002 (2004); Q.G. Huang and M. Li, Nucl. Phys. B 713, 219 (2005); J.P. van der Schaar, JHEP 0401, 070 (2004); G.L. Alberghi, K. Goldstein and D.A. Lowe, Phys. Lett. B 578, 247 (2004); G.L. Alberghi, D.A. Lowe and M. Trodden, JHEP 9907, 020 (1999); M. Fukuma, Y. Kono and A. Miwa, Nucl. Phys. B 682, 377 (2004); A. Tronconi, G. P. Vacca and G. Venturi, Phys. Rev. D 67, 063517 (2003); O. Elgaroy and S. Hannestad, Phys. Rev. D 68, 123513 (2003); E. Keski-Vakkuri and M.S. Sloth, JCAP 0308, 001 (2003); M.B. Einhorn and F. Larsen, Phys. Rev. D 68, 064002 (2003); S. Cremonini, Phys. Rev. D 68, 063514 (2003); G.L. Alberghi, R. Casadio and A. Tronconi, Phys. Lett. B 579 (2004) 1.

[3] G. L. Alberghi, R. Casadio and A. Tronconi, Phys. Rev. D 74, 103501 (2006).

[4] R. Brout and G. Venturi, Phys. Rev. D 39, 2436 (1989).

[5] B.S. DeWitt, Phys. Rev. 160, 1113 (1967); J.A. Wheeler, in Batelle rencontres: 1967 lectures in mathematics and physics edited by C. DeWitt and J.A. Wheeler, Benjamin, New York (1968).

[6] G.L. Alberghi, R. Casadio and A. Gruppuso, Phys. Rev. D 61 (2000) 084009.

[7] C.W. Misner, K.S. Thorne and J.A. Wheeler, Gravitation, W.H. Freeman and Co., San Francisco (1973).

[8] E. Anderson, Class. Quant. Grav. 24, 2935 (2007); Class. Quant. Grav. 24, 2971 (2007) and Class. Quant. Grav. 24, 2979 (2007)

[9] T.D. Lee, Particle Physics and Introduction to Field Theory (Harrwood, Chur, 1981); S. Gasiorowicz, Quantum Physics, (John Wiley \& Sons, New York, 1974); N.L. Balazs and B.K. Jennings, Phys. Rep. 104, 347 (1984).

[10] H.R. Lewis and W.B. Riesenfeld, J. Math. Phys. 10, 1458 (1969); X.-C. Gao, J.-B. Xu and T.-Z. Qian, Phys. Rev. A 44, 7016 (1991).

[11] C. Bertoni, F. Finelli and G. Venturi, Class. Quant. Grav. 13 2375, (1996).

[12] For a thorough discussion of the minisuperspace, the emergence of time and the WKB approximation, see Refs. [8].

[13] This is not necessarily the initial time of inflation. In fact, in Appendix A. we shall take initial conditions at $\tau=\tau_{0} \rightarrow-\infty$.

[14] We assume that during inflation $\mathcal{H} \sim 10^{-3} \ell_{\mathrm{P}}^{-1}$ [1].

[15] This $O\left(\ell_{\mathrm{P}}^{0}\right)$ equation reproduces the well-known result that conformally coupled scalar fields evolve freely on a cosmological background.

[16] More appropriately, one should then use a different degree of freedom to introduce the time.

[17] Note that there are typos in the numerical coefficients displayed in Ref. [3].

[18] We remark that using the Bessel functions as solutions $h_{1}$ and $h_{2}$ some cases, such as $\xi=1 / 4$ and $\mu=0$ cannot be analysed because the Wronskian $W$ becomes singular and the solution for $\sigma$ does not exist or is not unique.

[19] More precisely, these expressions hold for $z \gg \mid \nu^{2}-$ $1 / 4|=| \pm\left[(6 \xi-3 / 2)^{2}-\frac{\mu^{2}}{\mathcal{H}^{2}}\right]-\frac{1}{4} \mid$ and therefore, essentially, when $z \gg \frac{\mu^{2}}{\mathcal{H}^{2}}$ and $z \gg 1$. 